\title{
Effects of sleep deprivation on sustained attention in young adults
}

\section{IVAN ESAÚ PINTO VARGAS| STEFANE ALINE AGUIAR| JOSÉ ANGELO BARELA',2}

${ }^{1}$ Institute of Physical Activity and Sport Sciences, Cruzeiro do Sul University, São Paulo, SP, BRAZIL | ${ }^{2}$ Institute of Biosciences, São Paulo State University, Rio Claro, SP, BRAZIL

Correspondence to: José A. Barela, Instituto de Ciências da Atividade Física e Esportes - ICAFE, Universidade Cruzeiro do Sul, rua Galvão Bueno, 868 - São Paulo/SP, BRAZIL.

Email: jose.barela@cruzeirodosul.edu.br

\section{AT A GLANCE \\ This study revealed that sleep deprived young adults have their sustained attention capability decreased. Further, demonstrated that at the beginning of the task, subjects were able to maintain or even improve performance, nevertheless, subjects failed to maintain such performance after the fifth minute, showing a dramatic decrease in sustained attentional capability.}

\section{ABBREVIATIONS \\ CR Correct responses \\ TPT Tolouse-Piéron}

BACKGROUND: Sleep deprivation is common nowadays and considering that attention is important to our daily activities, it is important to compare the effects of sleep deprivation on sustained attention.

AIM: This study investigated the effects of sleep deprivation on sustained attention in young adults.

METHOD: Sixteen adults were evaluated both after a normal and after one night of sleep deprivation. Sustained attention was assessed through the Tolouse-Piéron Test (TPT), measuring the number of correct responses (CR), omissions, and attention coefficient, at first, fifth and tenth minutes of the test.

RESULTS: Results revealed a reduction in the number of $C R$ in the fifth compared to the first minute but only in the post-test session. The number of omissions increased in the fifth compared to the first minute in the post sleep deprivation. Attention coefficient decreased in the fifth and tenth compared to the first minute. CONCLUSION: Sleep deprivation deteriorates sustained attention, especially when adults are required to maintain attention for long periods.

PUBLICATION DATA

Received 27 Abr 2016

Accepted 03 Mai 2017

Published 07 Ago 2017

\section{INTRODUCTION}

Sleep restriction and even deprivation have become a common occurrence nowadays. Several changes in our daily routines such as long work journeys and social and family activities have led many to sleep less and usually in inappropriate conditions. Such dramatic change in sleep habits have influenced many to investigate the behavioral and functional effects of sleep restriction and deprivation on several activities, such as cognitive tasks (e.g. $\left.{ }^{1}\right)$, memory and decision making ${ }^{2}$ and postural control ${ }^{3}$.

Among the many functions impaired by sleep deprivation, attention has been widely investigated. In general, it has been demonstrated that sustained ${ }^{4}$ and selective attention ${ }^{1}$ are affected by sleep deprivation, leading to worsening of performance in adults. However, the effects of sleep deprivation are not so consistent regarding the use of a dual-task in

\begin{tabular}{l|l|l|l|l}
\hline $\begin{array}{l}\text { Vargas, Aguiar, } \\
\text { Barela }\end{array}$ & 2017 & VOL. 11 & N.1
\end{tabular}


Brazilian Journal of Motor Behavior

sleep-deprived adults. While decline in a primary task performance was observed (e.g.5), in other experimental conditions the addition of a secondary task even attenuated the negative sleep deprivation effects on the performance of the primary task (e.g. $\left.{ }^{6}\right)$. Despite this controversy, there is consensus that sleep deprivation and restriction impact the use of attention and such effects are quite dramatic to many of our activities.

Several methods and tools can be used to examine attention performance. From the wide variety of methods, some implies the use of sophisticated and expensive equipment such as the electrooculography ${ }^{7}$ and the eye tracking systems ${ }^{8}$. On the other hand, there are several similarly effective tests widely used to infer attentional capabilities that demand less technological resources. For example, simple reaction time test performed on a personal organizer has been used to infer sustained attention performance ${ }^{9}$. Through the use of such simpler and less demanding protocols, it has recently been demonstrated that vigilance performance is deteriorated by sleep deprivation ${ }^{10}$. Negative effects of sleep restriction on sustained attention and mood were also shown using an one-line system designed and programmed to delivery stimuli over the Internet ${ }^{11}$.

Another important test to measure attention resources is the Tolouse-Piéron Test (TPT), also referred as Tolouse-Pieron Scale (1986) ${ }^{12}$, and validated according to the Brazilian's conditions ${ }^{13}$. The TPT has been commonly used by psychologists to evaluate concentration range and to detect attention anomalies ${ }^{14}$, attention-deficit and hyperactivity disorder ${ }^{12}$, relationship between memory and attention deficits and emotional disorders ${ }^{15}$, sleep quality and attention ${ }^{16}$.

Evidence suggests that sleep deprivation affects performance especially when the task has a relatively long duration ${ }^{17}$. Since the TPT involves a ten-minute-period, it seems suitable to investigate the impact of sleep deprivation on attentional function. An important unresolved question is whether sleep deprivation affects performance in an attentional task throughout the entire time of the test or if changes in performance are observed only after the subject has been executing the task for a relatively long period. Therefore, the purpose of this study was to investigate the effects of one night of sleep deprivation on sustained attention in young adults in the first-, fifth- and tenth-minute periods.

\section{METHODS}

\section{Participants}

Sixteen healthy young adults (aging $23.6 \pm 6.0$ years) participated as volunteers in this study. Participants were undergraduate students, had normal or corrected-to-normal vision, and reported no diagnosed sleep disturbances. Prior to participation, volunteers signed a written consent form according to the procedures approved by the Institutional Ethics Committee.

\section{Procedures}

Sleep diaries were used to monitor participants' sleep schedules three days before the experiment and they were instructed to keep regular sleep during this period. Participants arrived at the laboratory in the morning, after a normal night of sleep for the first test session, which occurred between 8am and 11am. After this first test session, they were allowed to 
go about their daily activities and then returned to the lab approximately at 8pm, where they remained awake during the entire night. A second test session was performed after this sleep deprivation night, also between 8am and 11am. During the sleep deprivation period in the laboratory, volunteers engaged in activities that did not involve physical demands, such as reading and playing cards, and were provided with food. Alcohol and caffeine were prohibited. Two experimenters accompanied participants throughout the sleep deprivation night in order to assure that all the study compliances were fulfilled.

Sustained attention was evaluated in both test sessions, before and after the sleep deprivation night, through the Tolouse-Piéron Test (TPT). In order to do so, participants were provided with a sheet of paper containing 1200 squares, displayed in 30 lines and 40 columns, with lines from these squares pointing to different directions. At the top of the sheet, there are three target squares. The task was to identify among all 1200 squares which ones are identical to ones of the three target squares, that is, the squares which have the line pointing to the same direction to one of the three target squares. The participant was asked to scan as much as possible all of the 1200 squares and mark with a pencil every match square as fast and accurate as possible. The total duration of the test was 10 minutes. The TPT test was applied by the two experiments, previously trained, and they followed all the test application requirements.

In order to track the progression of the subjects' performance throughout the 10minute-period of the test, at every minute the experimenter gave a sign so participants would draw an "x" on the square that was being scanned at that exact moment. In this way, it was possible to screen the results from the entire test period (i.e., 10 minutes) as well for specific periods of the test (e.g., 1st - from the beginning to minute 1; 5th - from minute four to minute five; and 10th -from minute 9 to minute 10, as used in this study).

The responses were compared to a matrix in order to identify the correct responses, the errors and the omissions. Performance on the TPT was examined using three dependent variables: number of correct responses (CR), number of omissions and attention coefficient. The number of correct responses was the number of marks that correctly matched the target figures. The number of omissions corresponded to the number of correct figures presented in the sheet of paper but not marked by the subject. Finally, the attention coefficient was calculated according to the following:

$$
\text { AttentionCoefficient }=\frac{(\text { CR }- \text { Errors })}{(\text { Errors }+ \text { Omissions })}
$$

where, Errors represent the number of figures marked by the subject which did not match any of the target figures. All three dependent variables were calculated for the periods corresponding to the first, fifth, and tenth minutes of the test.

\section{Statistical Analysis}

Three analyses of variance (ANOVAs) having as factors the test, pre- and postsleep deprivation, and the minute within the test (1st, 5th, and 10th ), both factors treated as repeated measures, were conducted on each of the dependent variables: number of $C R$, number of omissions and attention coefficient. When necessary, Tukey HSD post hoc tests

\begin{tabular}{l|l|l|l|l}
\hline $\begin{array}{l}\text { Vargas, Aguiar, } \\
\text { Barela }\end{array}$ & 2017 & VOL. 11 & N.1
\end{tabular}


Brazilian Journal of Motor Behavior

were conducted and the effect size (Cohen's $d$ ) were presented, when comparisons reached significance. The significance level was set at 0.05 and all analyses were performed using the SPSS software

\section{RESULTS}

Sleep diaries showed that participants maintained regular sleep three days before the experiment (average of $7.9 \pm 0.7$ sleep-hour per night) and slept in average $8.1 \pm 0.6$ hours in the night prior to the experiment. According to sleep diaries, participants had been awake for 3.1 \pm 1.1 hours in the first test session (pre-sleep deprivation) and for $27.0 \pm 1.1$ hours in the second test session (post-sleep deprivation).

Figure 1 depicts the number of correct responses for the pre- and post-sleep deprivation tests and for the $1 \mathrm{st}$, 5 th, and 10 th minutes. ANOVA revealed test, $F(1,15)=9.17$, $p<0.009$, and minute effect, $F(2,30)=3.42, p<0.05$, and test and minute interaction, $F(1,21)=5.10, p<0.03$. The number of $C R$ was higher in the post- compared to the pre-sleep deprivation test $(d=1,36)$. None of the comparisons among the number of $C R$ in the $1 \mathrm{st}, 5$ th, and 10 th minutes reached significance. Post hoc tests only showed a reduced number of $\mathrm{CR}$ in the 5th compared to 1 st minute $(\mathrm{d}=-1,27)$ for the post-sleep deprivation test.

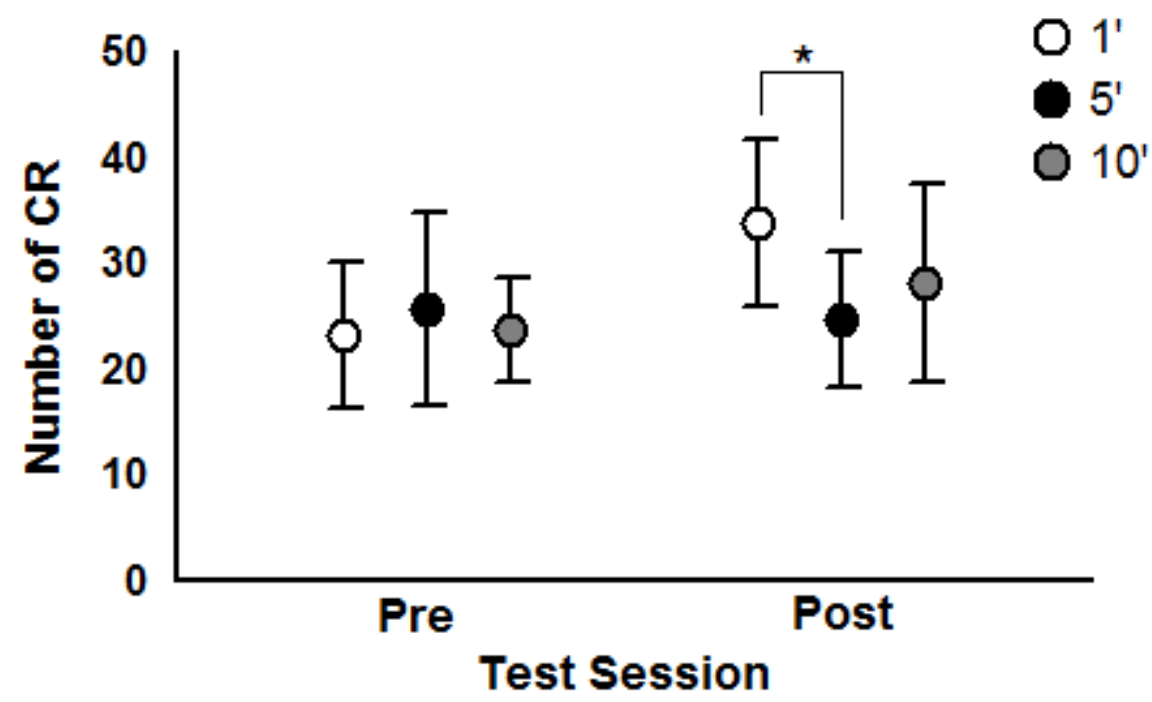

Figure 1. Average number of correct responses (CR) in the 1st, 5th and 10th minutes of the test during the pre- and post-sleep deprivation test sessions. Error bars represent standard deviation.

Figure 2 depicts the number of omission responses for the pre- and post-sleep deprivation tests and for the 1st, 5th, and 10th minutes. ANOVA revealed minute effect, $F(1,19)=6.04, p<0.02$, and test and minute interaction, $F(1,21)=3.80, p=0.05$, but no test effect, $F(1,15)=3.15, p>0.05$. Post hoc tests for the interaction showed that the number of omissions increased in the 5th compared to 1 st minute $(d=1,04)$, but only for the post-sleep deprivation test.

\begin{tabular}{l|l|l|l|l}
\hline $\begin{array}{l}\text { Vargas, Aguiar, } \\
\text { Barela }\end{array}$ & 2017 & VOL. 11 & N.1 &
\end{tabular}




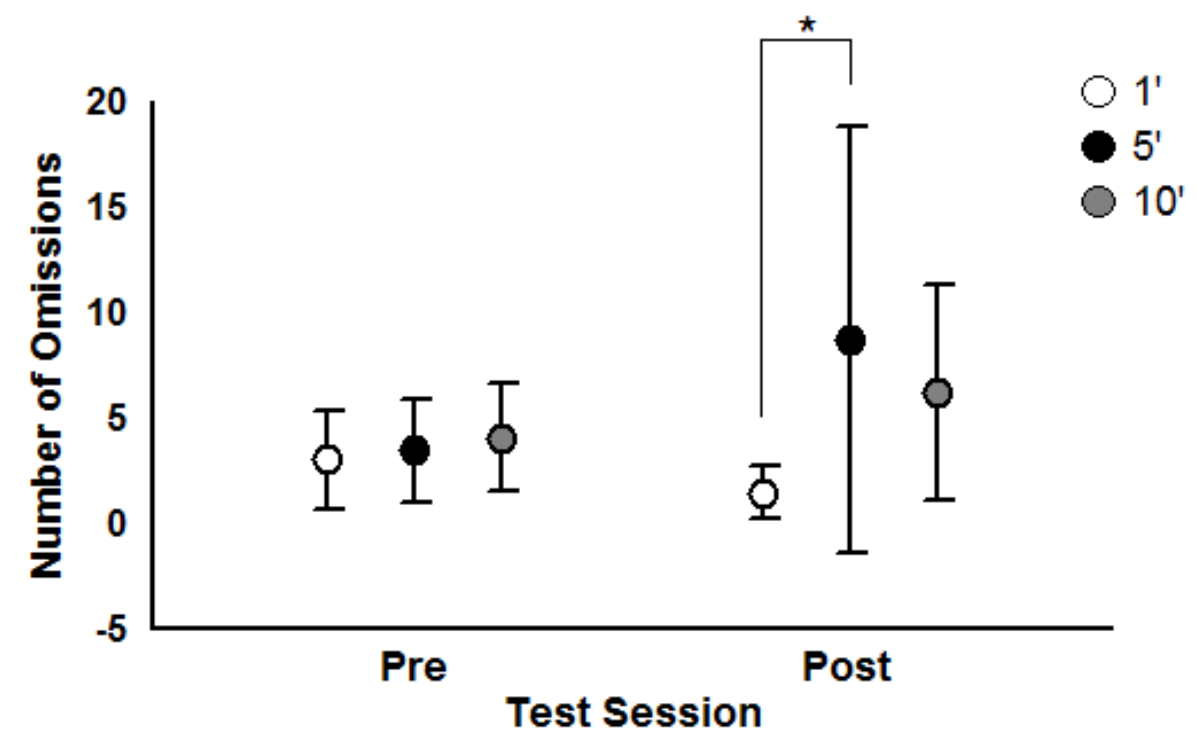

Figure 2. Average number of omissions in the 1st, 5th and 10th minutes of the test during the pre and post sleep deprivation test sessions. Error bars represent standard deviation.

Figure 3 depicts the attention coefficient for the pre- and post-sleep deprivation tests and for the 1st, 5 th, and 10 th minutes. ANOVA revealed minute, $F(2,30)=9.77, p<0.002$, but no test effect, $F(1,15)=1.23, p>0.05$, nor test and minute interaction, $F(2,30)=2.05, p>0.05$. Attention coefficient decreased in the 5th compared to the 1st minute $(d=-2,02)$ and 10th minutes compared also to the 1 st minute $(\mathrm{d}=-2,3)$.

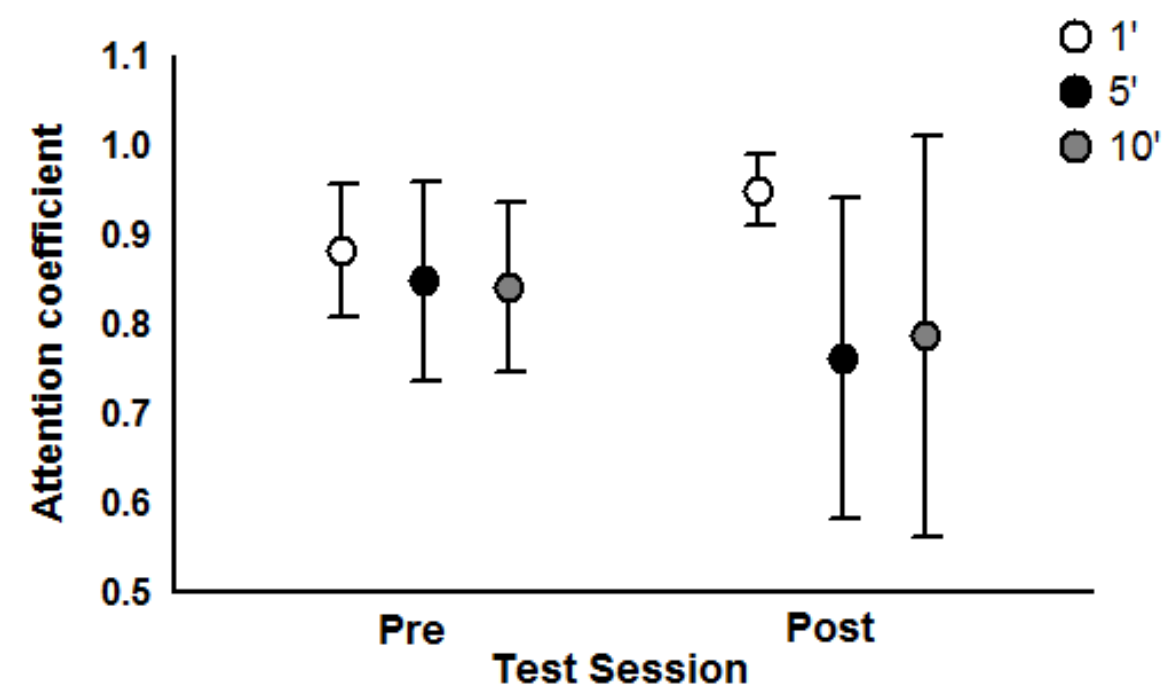

Figure 3. Average attention coefficient values in the 1st, 5th and 10th minutes of the test during the pre and post sleep deprivation test sessions. Error bars represent standard deviation

DISCUSSION

\begin{tabular}{l|l|l|l|}
\hline $\begin{array}{l}\text { Vargas, Aguiar, } \\
\text { Barela }\end{array}$ & 2017 & VOL. 11 & N.1
\end{tabular}


This study examined the effects of one night of sleep deprivation on sustained attention in young adults. Surprisingly, the results indicated that at the beginning of the attentional test young adults improved their performance after sleep deprivation. However, after the fifth minute of the test protocol, performance was considerably deteriorated. Taken together, these results suggest that sleep-deprived young adults seem to allocate more attention to perform a task, but soon such effort is not sustained to maintain initial performance for relatively long periods. Such difference in performance throughout the test is not observed prior to sleep deprivation.

After one night of sleep deprivation, at the beginning of the TPT, the number of correct responses increased. Such result is surprising since one could expect that after being sleep deprived, attention would be impaired, as previously observed 8,18 and even using the TPT ${ }^{19}$. However, performance of both correct and of omission responses was not sustained throughout the 10-minute-period of the test after sleep deprivation. The number of correct responses significantly decreased when measured at the fifth and the tenth minutes compared to the first minute. Similarly, number of omissions dramatically increased at the same fifth and tenth minutes compared to the first minute. A possible explanation for such results is that at the beginning of the test, young adults were able to use attentional resources and even improve their performance. Such suggestion seem to be in line with results showing no decrement in performance after sleep deprivation for certain tasks which is believed to depend on the nature of the cognitive task being performed 5 . For instance, in more complex tasks, such as logical reasoning (grammatical transformation), it was observed an increasing of cerebral responses for sleep-deprived adults, interpreted that sleep-deprived adults would be compensating for the deleterious effects of the lack of sleep in order to try to maintain performance ${ }^{20}$.

In this way, a conscious cognitive effort employed by adults at the first minutes of the task would be a possible explanation of why performance was maintained or improved after sleep deprivation. The novelty of our results is that such effort was shown to fail after a relatively long period of sustained performance, which led to increased number of omissions and fewer correct responses. In this case, such performance detriment was observed after the fifth minute of the task.

Several studies have suggested that sustained attention after sleep deprivation is affected21-23. Our results clearly showed that despite the effort and even performance improvement at the beginning, after a period of time (at least after a five-minute period), young adults are not capable of sustaining the same level of attention when sleep-deprived. Moreover, the deterioration of performance was quite dramatic, as observed with the significant increase in omissions observed at the fifth- and tenth-minute periods. Although attention coefficient did not reach statistical significance (test and minute interaction), it was also observed a tendency of decrease in attention coefficient at the fifth and tenth minutes. Again, no changes were observed in any of the three variables (correct responses, number of omissions, and attention coefficient) prior to the sleep deprivation.

Sustained attention is important in many of our daily activities and, in many cases that one is not capable of maintaining attention for a period of time, the consequences might be undesirable. This becomes extremely important when considering that many tasks commonly performed by sleep-deprived individuals have high level of attentional requirements; examples include driving and patient care related tasks performed by doctors 
Brazilian Journal of Motor Behavior

on long work-shifts 24,25 . With this regard, although the TPT shows limitations and still needs to be further used, our results indicated that the TPT could be considered as a simple but important measure of attentional functions of individuals under sleep deprivation. However, the need for the assessment to account for variations in performance throughout the 10 minute period of the test instead of using just standardized global variables must be emphasized, as our results demonstrate that sleep deprivation effects were observed only after the period of 5 minutes of task onset.

\section{ACKNOWLEDGMENTS}

This study was supported by CAPES/PROSUP, Brazilian Federal Government.

\section{REFERENCES}

1. Chee MW, Tan JC, Parimal S, Zagorodnov V. Sleep deprivation and its effects on objectselective attention. Neuroimage. 2010;49(2):1903-10.

2. Killgore WD. Effects of sleep deprivation on cognition. Prog Brain Res. 2010;185:105-29.

3. Aguiar SA, Barela JA. Sleep deprivation affects sensorimotor coupling in postural control of young adults. Neurosci Lett. 2014;574:47-52.

4. Patel M, Gomez S, Berg S, Almbladh P, Lindblad J, Petersen H, et al. Effects of 24-h and 36-h sleep deprivation on human postural control and adaptation. Experimental brain research. 2008;185(2):165-73.

5. Robillard R, Prince F, Boissonneault M, Filipini D, Carrier J. Effects of increased homeostatic sleep pressure on postural control and their modulation by attentional resources. Clin Neurophysiol. 2011;122(9):1771-8.

6. Gazes Y, Rakitin BC, Steffener J, Habeck C, Lisanby SH, Butterfield B, et al. Dual-tasking alleviated sleep deprivation disruption in visuomotor tracking: an fMRI study. Brain Cogn. 2012;78(3):248-56.

7. Baumann $\mathrm{O}$, Greenlee MW. Effects of attention to auditory motion on cortical activations during smooth pursuit eye tracking. PLoS One. 2009;4(9):e7110.

8. Bocca ML, Denise P. Total sleep deprivation effect on disengagement of spatial attention as assessed by saccadic eye movements. Clin Neurophysiol. 2006;117(4):894-9.

9. Taillard J, Moore N, Claustrat B, Coste O, Bioulac B, Philip P. Nocturnal sustained attention during sleep deprivation can be predicted by specific periods of subjective daytime alertness in normal young humans. J Sleep Res. 2006;15(1):41-5.

10. Roca J, Fuentes LJ, Marotta A, Lopez-Ramon MF, Castro C, Lupianez J, et al. The effects of sleep deprivation on the attentional functions and vigilance. Acta Psychol (Amst). 2012;140(2):164-76.

11. Kahn M, Fridenson S, Lerer R, Bar-Haim Y, Sadeh A. Effects of one night of induced nightwakings versus sleep restriction on sustained attention and mood: a pilot study. Sleep Med. 2014;15(7):825-32. 
12. Mokntari S, Behpazhoh A, Afrooz GA, Ebrahimi N, Samira S. Effectiveness of sensory integration method on improving children's attention with attention-deficit and hyperactivity disorder. Journal of Social Issues \& Humanities. 2014;2(Issue 8).

13. Montiel JM, Figuereido ER, Lutosa SD, Dias NM. Evidência de validade para o Teste de Atenção Concentrada Toulouse-Piéron no contexto de trânsito. Psicologia: Pesquisa \& Trânsito. 2006;2:19-27.

14. Mazon J. The influence of thermal discomfort on the attention index of teenagers: an experimental evaluation. Int J Biometeorol. 2014;58(5):717-24.

15. Fioravanti M, Carbone G, Galli N, Piccirilli E, Pierucci P. Relationship between memory, attention, and depression in chronic cerebro-vascular patients. J Neurol Sci. 2007;257(1-2):1559.

16. Pacheco AA. Relation between sleep quality and attention in students of business administration. Biological Rhythm Research. 2014;45(1):131-42.

17. Martella D, Casagrande M, Lupianez J. Alerting, orienting and executive control: the effects of sleep deprivation on attentional networks. Experimental brain research. 2011;210(1):81-9.

18. Chee MW, Tan JC, Zheng H, Parimal S, Weissman DH, Zagorodnov V, et al. Lapsing during sleep deprivation is associated with distributed changes in brain activation. The Journal of neuroscience : the official journal of the Society for Neuroscience. 2008;28(21):5519-28.

19. Sanches I, Teixeira F, dos Santos JM, Ferreira AJ. Effects of acute sleep deprivation resulting from night shift work on young doctors. Acta medica portuguesa. 2015;28(4):457-62.

20. Drummond SP, Brown GG, Salamat JS, Gillin JC. Increasing task difficulty facilitates the cerebral compensatory response to total sleep deprivation. Sleep. 2004;27(3):445-51.

21. Chua EC, Yeo SC, Lee IT, Tan LC, Lau P, Cai S, et al. Sustained attention performance during sleep deprivation associates with instability in behavior and physiologic measures at baseline. Sleep. 2014;37(1):27-39.

22. Sadeh A, Dan O, Bar-Haim Y. Online assessment of sustained attention following sleep restriction. Sleep Med. 2011;12(3):257-61.

23. Gunzelmann G, Gross JB, Gluck KA, Dinges DF. Sleep deprivation and sustained attention performance: integrating mathematical and cognitive modeling. Cogn Sci. 2009;33(5):880-910. 24. Philip P, Akerstedt T. Transport and industrial safety, how are they affected by sleepiness and sleep restriction? Sleep Med Rev. 2006;10(5):347-56.

25. Jackson ML, Croft RJ, Kennedy GA, Owens K, Howard ME. Cognitive components of simulated driving performance: Sleep loss effects and predictors. Accid Anal Prev. 2013;50:438- 
Brazilian Journal of Motor Behavior

Citation: Vargas IEP, Aguiar AS, Barela JA Effects of sleep deprivation on sustained attention in young adults. BJMB. 2017: 12(1): 1-10.

Editor: Guilherme Menezes Lage, Universidade Federal de Minas gerais, MG, Brasil.

Copyright: @ 2017 Vargas, Aguiar, Barela and BJMB. This is an open-access article distributed under the terms of the Creative Commons Attribution-NonCommercial-NoDerivatives 4.0 International License which permits unrestricted use, distribution, and reproduction in any medium, provided the original author and source are credited.

Funding: This study was supported by CAPES/PROSUP, Brazilian Federal Government.

Competing interests: The authors have declared that no competing interests exist.

Download: http://socibracom.com/bjmb/index.php/bjmb/issue/archive 